\title{
Improvement the System to Fold Laundry that has be Hung up
}

\author{
Takumi Saruhashi ${ }^{\mathrm{a}}$, Ryunosuke Miyamoto ${ }^{\mathrm{b}}$, Shenglin $\mathrm{Mu}^{\mathrm{c}}$, Takaaki Akimoto ${ }^{\mathrm{a}}$, Yuhki Kitazono ${ }^{\mathrm{a}}$ \\ ${ }^{a}$ National Institute of Technology, Kitakyushu College,5-20-1 Shii, Kokuraminami-ku, Kitakyushu-city, Fukuoka \\ 802-0985, Japan \\ ${ }^{\mathrm{b}}$ Molex Japan Co., Ltd., 1-5-4 Hukamihigashi, Yamati-city, Kanagawa 242-8585, Japan \\ ${ }^{\mathrm{c}}$ National Institute of Technology, Hiroshima College, 4272-1, Higashino, Osakikamijima-cho, Toyota-gun, Hiroshima \\ 725-0231, Japan \\ *Corresponding Author:kitazono@kct.ac.jp
}

\begin{abstract}
In this paper, we discuss the laundry folding system that we have developed. This system can take laundry off the hanger from laundry and fold laundry in automatically. After you take the laundry out of washing machine and put it on a hanger rack with the hanger, our system takes each hanger from the hanger rack, moreover detects whether the laundry is dry or not. If laundry is dry, hanger is removed from laundry. Thereafter laundry is folded by the device in the system. Therefore, all things that user have to do is only hanging washed laundry on a hanger. However, the prototype of the system could fold only a T-shirt. Hence we have improved the device of folding laundry. In this time, we have made the device fold some kinds of laundry; shirt, trouser, and skirt. As a result, the system has become able to fold some kind of laundry.
\end{abstract}

Keywords: folding the laundry, robot, folding device, Arduino, motor.

\section{Introduction}

Housework is one of tough jobs. Not only that, we must do it every day. We can't live comfortably without doing housework. In the case of a single life, you have to do that as well as your work. On the other hand, if you have a large family, amount of housework becomes enormous. There are a great number of household tasks such as cleaning the room, washing the dishes, and doing the washing (1) (2). Previously, housework has been done manually. However, as a result of appearance of Dishwasher, Vacuum cleaner, and Washing machine, we can conveniently save trouble. Nevertheless, the machine which folds the laundry automatically has not prevailed among the ordinary homes. Even today, folding the laundry is a manual labor.

In 2015, the laundry folding robot: "Laundroid" was developed by Seven Dreamers Laboratories, Inc., Panasonic Corporation, and Daiwa House Industry Co.,Ltd. Though the robot has ability to fold many kind of clothes, it takes approximately 10 minutes to fold one T-shirt, and it doesn't consider whether the laundry has already dried or not. Due to that, user should check whether the laundry is dry when he takes the laundry in. Folding laundry in automatically has been studied by some researcher ${ }^{(3)}$ (4). However, the laundry is dried in the laundry dryer, so hanging the laundry to dry process is ignored in previous studies. When we take in account the percentage of households owning a laundry dryer, hanging out the laundry to dry is yet main means to dry the laundry ${ }^{(5)(6)}$. Addition to this, some clothes can't be dried in the laundry dryer because they shrink in the heat of the dryer. Also there are some attempts to keep a proper humidity of the room by hanging out the laundry to dry in the room to prevent the room from drying ${ }^{(7)(8)}$. If we hanged the laundry out, we have to detect whether the laundry is dried or not when we take the laundry in. After that, we have to remove the hanger from laundry to fold laundry. However, these processes of folding laundry are not much studied.

From such background, we had developed the system to fold laundry that state hang out. The developed system could detect whether the laundry is dry or not. After detecting that the laundry is already dry, the system removes the hanger from the laundry and folds the laundry. It took time about 1 minute and 30 seconds to fold a laundry. However, the prototype of the system could fold only a T-shirt. Therefore, we had improved the folding laundry device to deal with some kinds of laundry. 


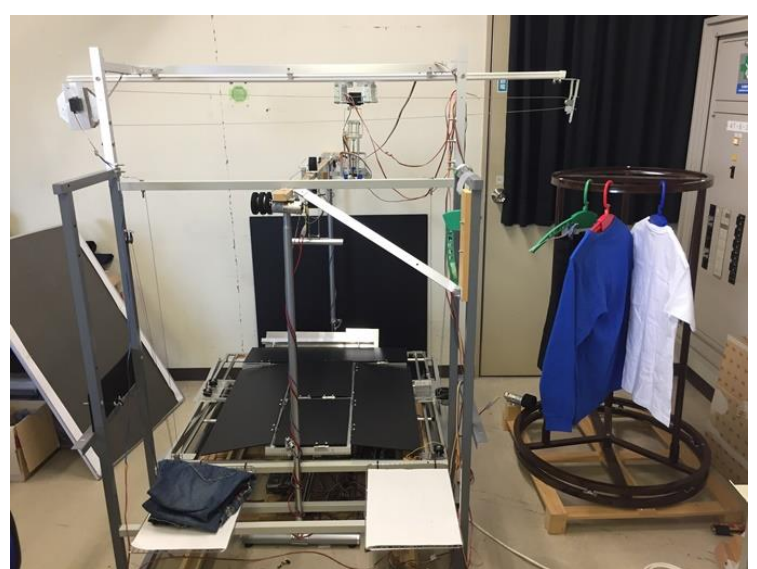

Fig. 1. Overall view of the system.

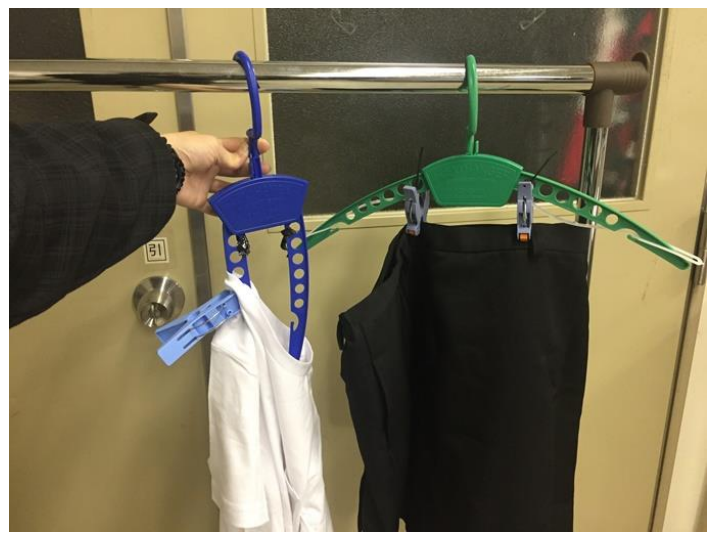

Fig. 2. The hanger and clothespins.

\section{Construction of System}

\subsection{System overall view and the operating procedure}

The overall view of the system is shown in Fig. 1. The rotary hanger rack part is shown in right side in the Fig. 1. The user hangs laundry on the rotary hanger rack. We use the clothes pin (NSA-37 by NAITO-KOGYOSHO, INC.) and hanger (DFQ011 by DAISAKU-SHOJI, INC) as shown in Fig. 2 to hang laundry. We pinch the laundry to clothespins of the hanger. If the laundry has a sleeve such as a shirt, we hang the laundry to the hanger in normally. So, the sleeve is put through by the wings of the hanger. In Fig. 2 , the tip of the clothes pin is roller-like shape. The laundry that is pinched by the clothes pin can be taken easily by pulling the laundry or hanger. Also the hanger has special structure. Wings of the hanger are bent if the button of the hanger handle is pushed. Therefore, we can remove the

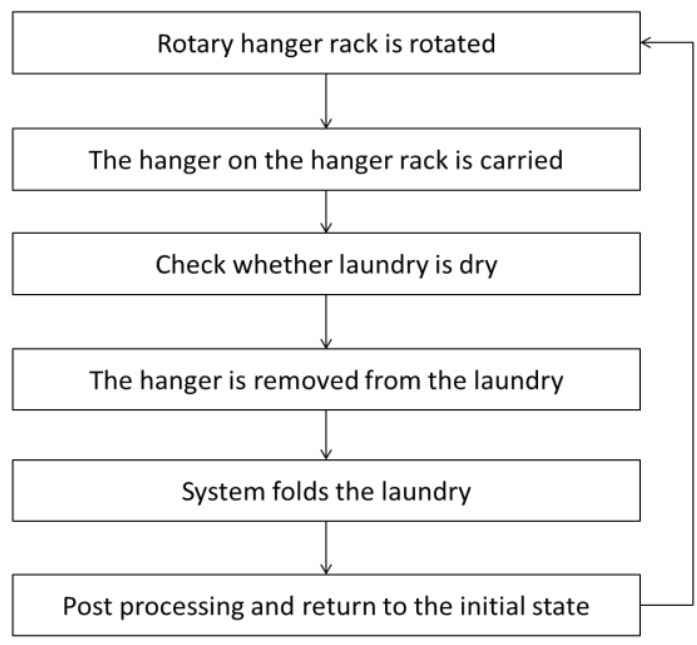

Fig. 3. System operation procedure.

hanger from laundry neckline.

Folding laundry device part is shown the left side in the Fig. 1. This device is the main part of the system. It detects dryness of laundry, removes hanger from laundry, and folds laundry.

The behavior of the system is shown in Fig. 3. By using motors and operating the respective device, these operations are realized. Also state of each device is detected by sensors and motors, and they are controlled by microcomputer. AC100V is used for the power supply of the system. It is converted to DC5V and DC $12 \mathrm{~V}$ by the power strip. The converted voltage is supplied to each device.

\subsection{Improved laundry folding device}

The system controls some device to fold laundry in automatically. The behaver of the system can roughly divide into two parts: carrying laundry process and folding laundry process. In carrying hanger process, the system makes the laundry moves from rotary hanger rack. Thereafter, the system removes hanger from laundry. Finally in this process, the laundry placed on the laundry folding device. After that, the system runs the folding process and controls laundry folding device. However, it device had some problem.

Therefore, we improved the folding laundry device as shown in Fig. 4 and Fig. 5. Fig. 4 is previous device in our study. This device could fold laundry. However, it could only fold in two as for the longitudinal of laundry. Addition to this, the device size is too small to fold large laundry well. The size of device is about 0.7 meter square. The 


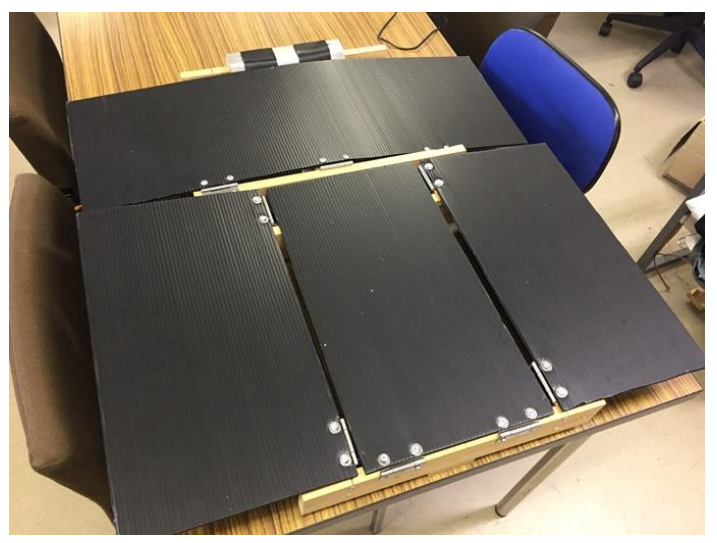

Fig. 4. Previous folding laundry device.

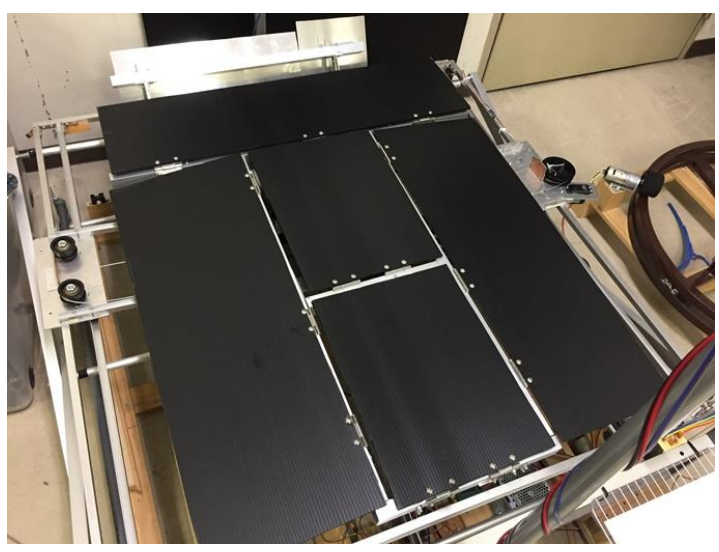

Fig. 5. Improved folding laundry device.

length of device is shorter than laundry like a trouser. Hence, the device couldn't fold laundry well.

The improved device can fold laundry better than previous device. The improved device is 1.2 meter length and 0.75 meter width. Moreover, it can fold laundry in three. In new device, laundry that has sleeve like a T-shirt is folded as shown in Fig. 6. The laundry that has no sleeve is folded as shown in Fig. 7.

\section{Devices in the System}

\subsection{Devices which are operated by the system in carrying laundry process}

There are three devices that are run carrying laundry process. First one is rotary hanger rack. It is rotated by the DC motor to move the laundry on the hanger rack. As a result, the laundry is carried to the lift as shown in Fig. 8. We use this lift to carry the laundry with the hanger. The sensor attached lift and measures the distance between the arm of lift and the sensor. When the laundry moved there by the rotary hanger rack, the lift catches the hanger using the
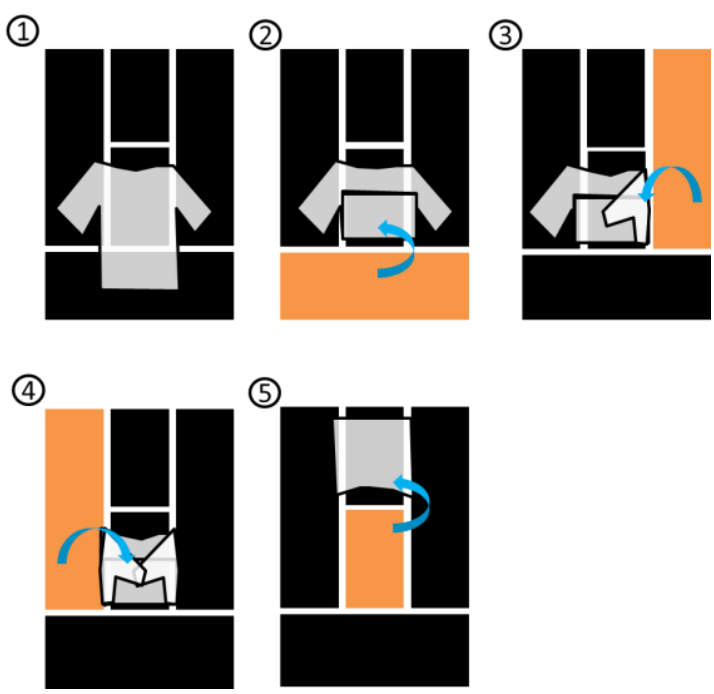

Fig. 6. Step of folding instrument about laundry that has sleeve.
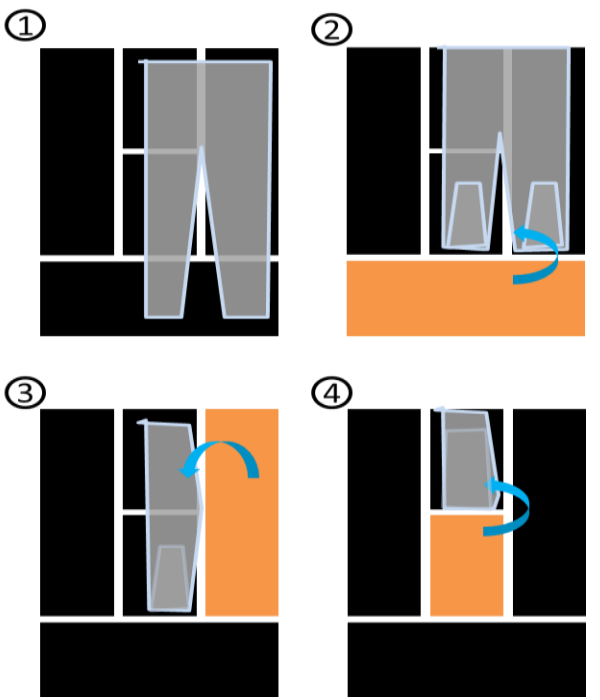

Fig. 7. Step of folding instrument about laundry that has no sleeve.

arm. There after the lift carry the laundry to folding laundry device part. In the middle of this process, the system captures the image of laundry using a web camera. After that, the system distinguishes whether the laundry has sleeve or not. We used raspberry pi and OpenCV library to distinguish laundry. After the lift carrying the laundry, the hanger mobile device as shown in Fig. 9 is operated. We use this device to move the laundry above the folding laundry device. When the laundry is carried by the lift, hanger mobile device catches the hanger using the arm and moves along the rail. The rail is laid above folding laundry device. In other words, the laundry is moved above folding laundry device to place on it. After that, the system run the folding laundry process. 


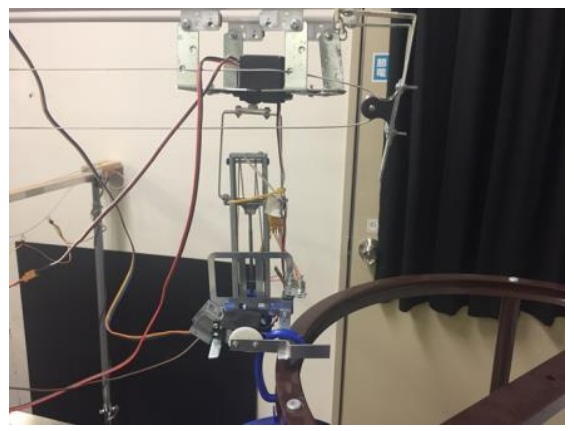

Fig. 8. Appearance of the Lift.

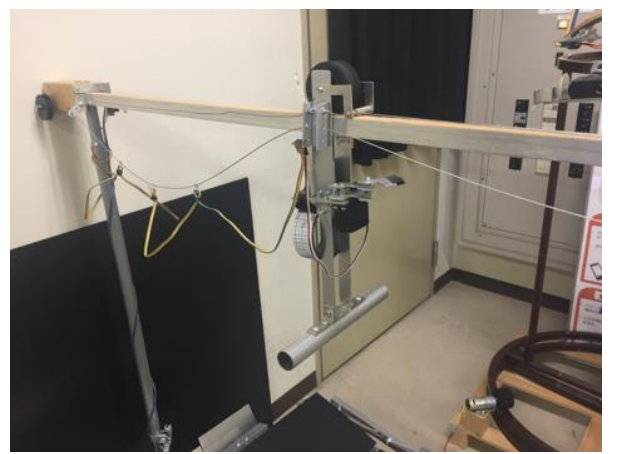

Fig. 9. Appearance of the Hanger mobile device.

\subsection{Devices which are operated by the system in folding laundry process}

In folding laundry process, the system controls folding laundry device behavior and it position. The folding laundry device consists of eight servo motor, one distance measuring sensor and Arduino. The servo motor is attached in panel of folding device as shown in Fig. 10. Panels can fold laundry by rotating servo motor that attached each panel. Also the arm and distance measuring sensor are attached in the panel as shown in Fig. 11. The sensor detects laundry that is moved by hanger mobile device. In Fig. 11, when the laundry is moved to between the aluminum bar and the aluminum plate, the sensor detects the laundry. Thereafter, the arm of device is closed to fix laundry. As shown in Fig. 12, the laundry is sandwiched between the aluminum bar and aluminum plate. After that, the laundry is stretched above the folding device. Thereafter, the hanger mobile device removes the hanger from laundry. As a result, the laundry is placed on folding device as shown in Fig. 13. In the middle of this process, the folding device is raised by system to place laundry properly. The horizontal position of device is controlled by servo motor as shown in Fig. 14. The folding device can move horizontal direction and fold laundry as shown in Fig. 6 and 7.

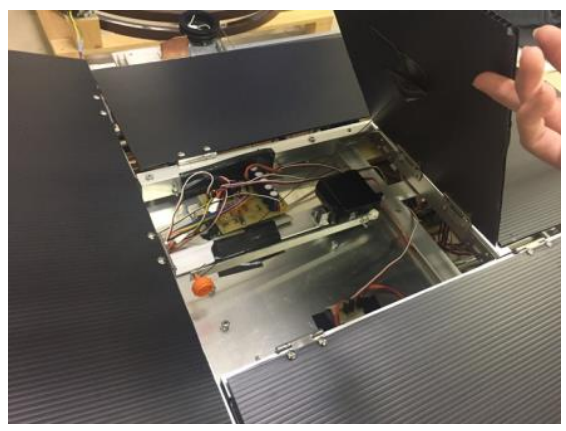

Fig. 10. Inside of the folding device panel.

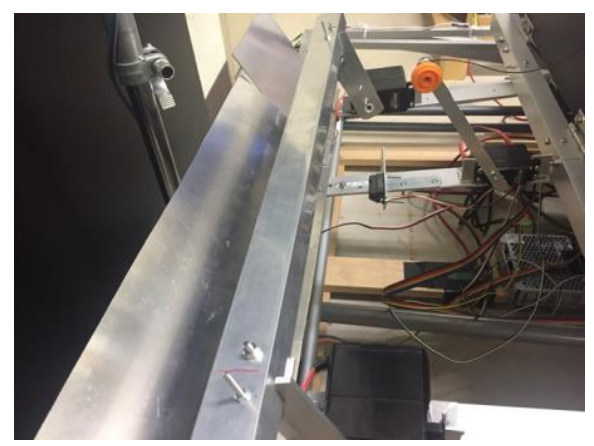

Fig. 11. The arm and distance measuring sensor are atthaced in the folding device.

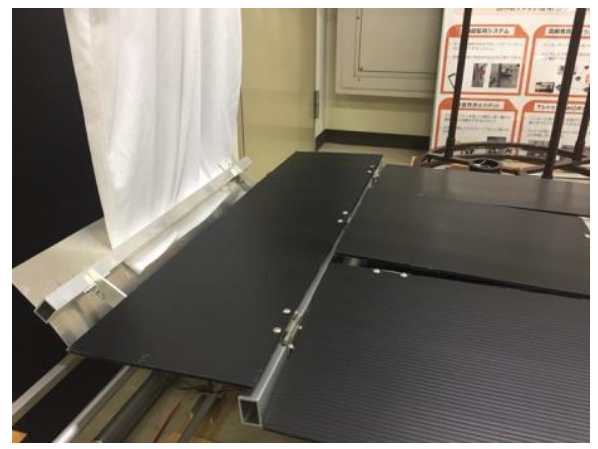

Fig. 12. The laundry is fixed by aluminum bar and plate.

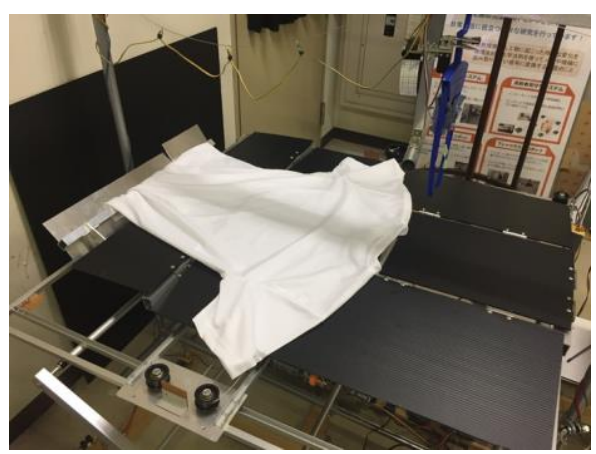

Fig. 13. The laundry is placed on device. 


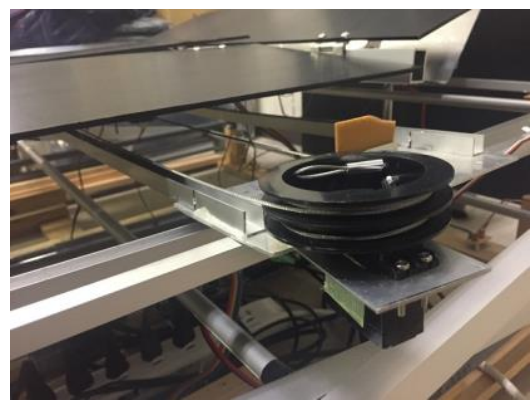

Fig. 14. This servo motor controls folding device position.

Table 1. The size of laundry.

\begin{tabular}{|c|c|}
\hline Clothes & Size (Japan) \\
\hline Short-sleeved shirt & L \\
\hline Long-sleeved shirt & L \\
\hline Skirt & S \\
\hline Trouser & M \\
\hline
\end{tabular}

\section{Experiment}

We checked the performance of the folding laundry device that we have improved. We have made the device fold short-sleeved shirt, long-sleeved shirt, skirt three quarter length trouser. The size of clothes is shown as Table 1.

\section{Result of Experiment}

The actual condition of the experiment is shown in Figs. 15, 16, and 17. As a result of experiment, the system could fold each laundry. In fact, the folding device could fold not only three quarter trouser but also full length trouser. However, the system couldn't place the full length trouser on folding device correctly. The reason for it is that although the folding device can fold the trouser well, the height of folding part in the system is shorter than trouser length.

\section{Conclusions}

Although the folding device is improved and it can fold some kind of laundry, we must improve or developed other devices for the purpose of folding long length clothe. Future issue of our study is that we make the system so that it can fold large size of laundry.

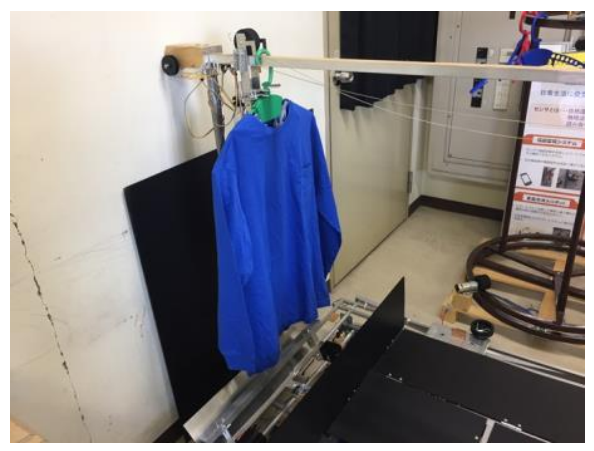

(a) Shirt is carried above the device.

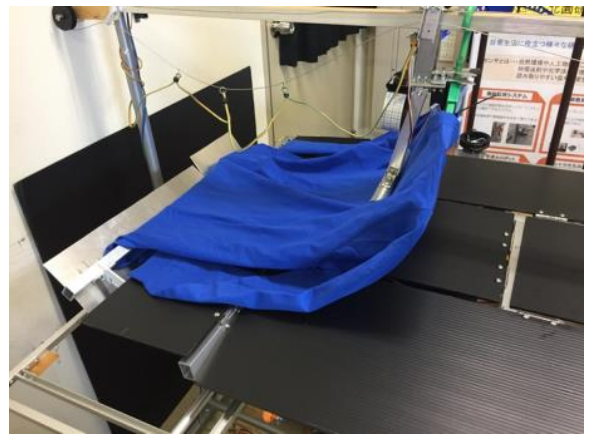

(b) Shirt is stretched.

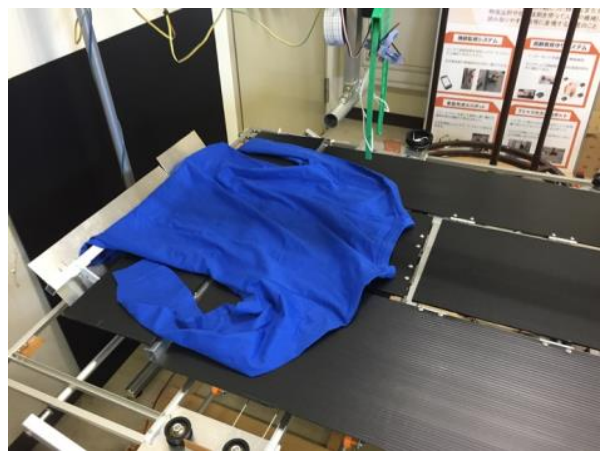

(c) The hanger is removed.

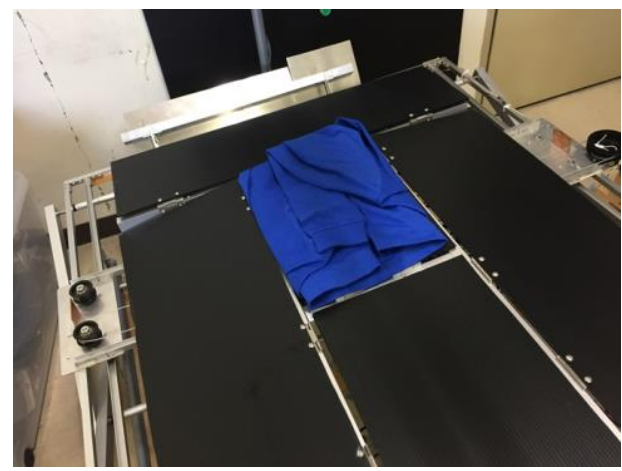

(d) Device folds the shirt.

Fig. 15. Folding shirt. 


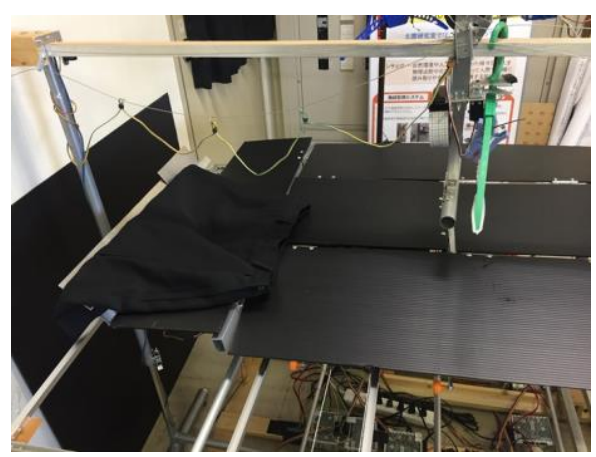

(a) Skirt is placed on the device.

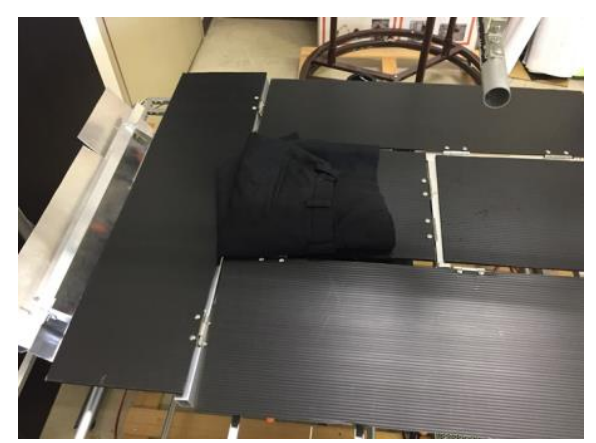

(b) Device folds skirt.

Fig. 16. Folding skirt.

\section{References}

(1) Kabata Shinichiro : "Technological Progress of Washing Machine", Journal of Japan Society of Mechanical Engineers, Vol. 115, No. 1120, pp. 146-147, 2012

(2) Nishiwaki S. : "Tecqnology Trend of Washing Machine", Journal of Japan Society of Mechanical Engineers, Vol. 115, No. 1120, pp. 146-147, 2012

(3) Hamajima Kyoko, Kakikura Masayoshi : "Planning Strategy for Task of Utangling Laundry - Isolation of Clothes from Washed Mass -", JRM, Vol. 10, No. 3, pp. 244-251, 1998

(4) Kaneko Manabu, Kakikura Masayoshi : "Study on Handling Clothes (Task Planning of Deformation for Unfolding Laundry)" JRM, Vol. 15, No. 4, pp. 406-415, 2003

(5) Fukuda Eiko : "Survey of Home Washing", The journal of Wayo Women's University, Vol. 43, pp. 85-98, 2003

(6) Koitabashi Emiko, Okita Fumiko : "The Actual Conditions of Washing Work/Working Space and Problems to Be Solved : From an Investigation Carried

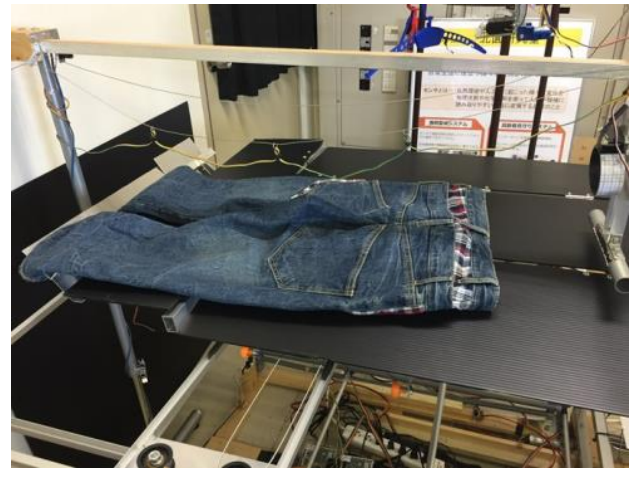

(a) Trouser is placed on the device.

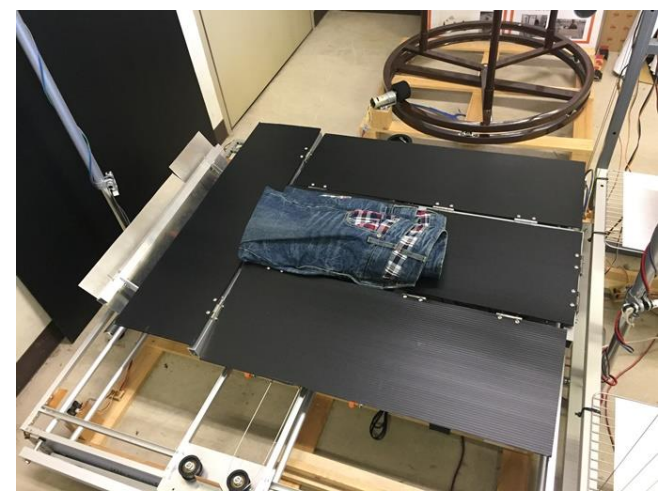

(b) Device folds trouser

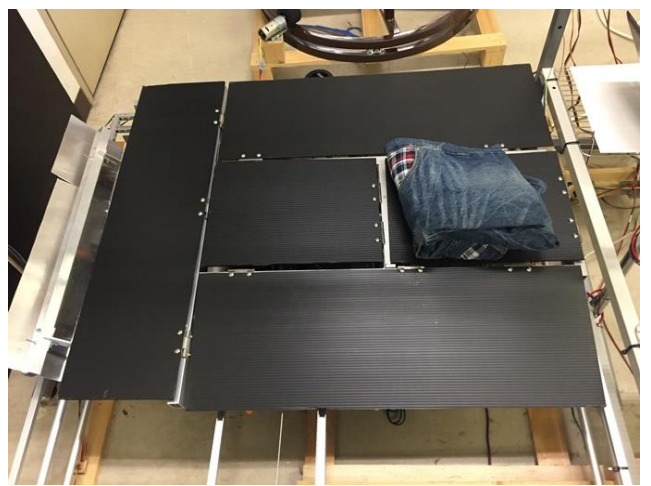

(c) Finished folding trouser.

Fig. 17. Folding trouser.

Out in 2000 and in 2008", Memoirs of the Japan Women's University. Faculty of Home Economics, Vol. 57, pp. 95-106, 2010

(7) Kazue Shoushi, Sato Manami : “41159 Study on control methods of relative humidity in a room to use desorption from finishing material and clothes : Part 1 An evaluation of hygrothermal distribution by actual measurement", Summaries of technical papers of Annual Meeting Architectural Institute of Japan. D-2, Environmental engineering II, Heat, moisture, thermal 
comfort, natural energy, air flow, ventilation, smoke exhaustion, computational fluid dynamics, indoor air quality heating, cooling and air-conditioning heat and cold sources, piping systems application of building services 2008, pp. 317-318, 2008

(8) Kazue Shoushi, Sato Manami : "41160 Study on control methods of relative humidity in a room to use desorption from finishing material and clothes: Part 2 An numerical analysis of hygrothermal distribution", Summaries of technical papers of Annual Meeting Architectural Institute of Japan. D-2, Environmental engineering II, Heat, moisture, thermal comfort, natural energy, air flow, ventilation, smoke exhaustion, computational fluid dynamics, indoor air quality heating, cooling and air-conditioning heat and cold sources, piping systems application of building services 2008, pp. 319-320, 2008 